\title{
Results of quality control of large size GEM detectors based on the Korean GEM foil for the future upgrades of the CMS muon system
}

\author{
Inseok Yoon* on behalf of the CMS Collaboration \\ The Research Institute of Basic Science, Seoul National University, 1, Gwanak-ro, Gwanak-gu, \\ Seoul, 08826, Republic of Korea \\ E-mail: inseok.yoon@ern.ch
}

\begin{abstract}
Since a luminosity upgrade of the LHC is scheduled, an upgrade of the CMS experiment is also planned. As part, detectors based on the gas electron multiplier (GEM) technology will be installed in the CMS endcaps. The Korean CMS group is one the group responsible for the large GEM foil production. As the foils are produced by a new manufacturer, Mecaro, an extensive quality check was planned and implemented. Several CMS GE1/1 GEM detectors with GEM foils produced by Mecaro have been assembled and the properties of those detectors have been measured. According to the measurements, the Mecaro foils are as good as CERN foils in terms of the effective gas gain, the uniformity of the gain, the rate capability and the radiation hardness. The results of the validation are presented.
\end{abstract}

EPS-HEP 2017, European Physical Society conference on High Energy Physics

5-12 July 2017

Venice, Italy

\footnotetext{
* Speaker.
} 


\section{KCMS \& Mecaro consortium}

Since a luminosity upgrade of the LHC (HL-LHC) is scheduled [1], an upgrade of the CMS detector is also planned to maintain the detector performances. As a part of the upgrade of the CMS muon system, three detector stations based on the gas electron multiplier (GEM) technology will be installed in the CMS endcaps [2][3]. These stations are called ME0, GE1/1, and GE2/1, numbered from the interaction point, respectively.

The Korean CMS group (KCMS) has created a consortium with Mecaro, the Korean company producing components and materials for semiconductor production, in order to have a second supplier of large size GEM foils. For a long time, the CERN micro pattern technologies laboratory has been the only supplier of large size GEM foils. However, CERN is not a mass production facility. Thus CERN alone could not have satisfied the GEM foil production demands. The shortage of GEM foils is a significant problem for the CMS upgrade. Consequently, it is necessary to find a second supplier. It is for this reason that the consortium has been made. It is the goal of the consortium to contribute towards the CMS phase-II upgrade and the other experiments by supplying large size GEM foils. Mecaro GEM foils are supposed to be used for the GE2/1 and ME0 detector assembly.

\section{GEM foil production at Mecaro}

Mecaro produces large size GEM foils with standard geometry by using a double-mask technique. The diameter of the inner (outer) holes is $50(70) \mu \mathrm{m}$ with a pitch of $140 \mu \mathrm{m}$. Mecaro is the world's first and only large size GEM foil supplier that uses a double-mask technique. As the technique is used, the shape of the hole is symmetrically biconical. The double-mask technique is chosen for faster production because much simpler production processes become possible compared to a single-mask technique. Thanks to the double-mask technique and automated machineries, a faster production rate is achieved. The current production rate is about 10 foils per week. The rate is expected to increase if production processes become stable.

The maximum GEM foil size producible by Mecaro is $1300 \mathrm{~mm} \times 600 \mathrm{~mm}$. The maximum foil size is set by the machinery which handles the alignment of masks and the exposure of ultraviolet light. As Mecaro is using the double-mask technique, mask alignment becomes extremely crucial. It was checked that the residual misalignment is less than $3 \mu \mathrm{m}$.

\section{Quality validation with the CMS GE1/1 detector}

The intensive quality validation of Mecaro GEM foils is being carried out as the foils are planned to be used for the CMS upgrade. Firstly optical inspections and leakage current measurements, which are established for the CMS GE1/1 project, were performed for the quality control (QC) of the Mecaro GEM foils. After the GEM foils passed the QC, CMS GE1/1 detectors were assembled with Mecaro GEM foils and the properties of the detectors were measured. CMS GE1/1 detectors are triple-GEM detectors filled with an $\mathrm{Ar} / \mathrm{CO}_{2}(70 \% / 30 \%)$ mixture. High voltage is supplied to the detector elements through an in-house designed voltage divider.

The first measured properties were the effective gas gain curves and the gain uniformities. Fig. 1 shows the gain curves and the gain distributions for the uniformity measurement. To check 


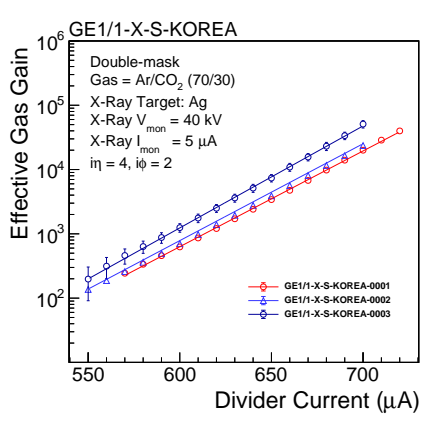

(a)

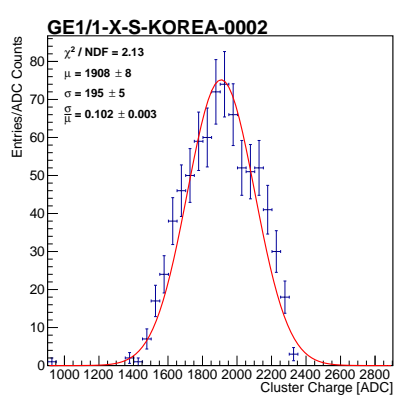

(c)

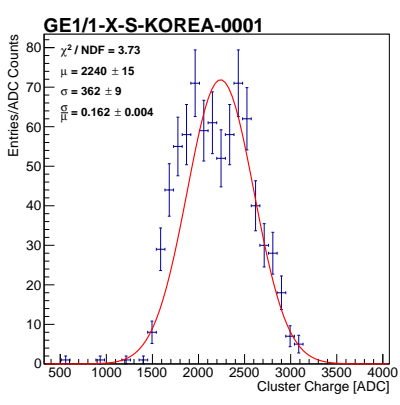

(b)

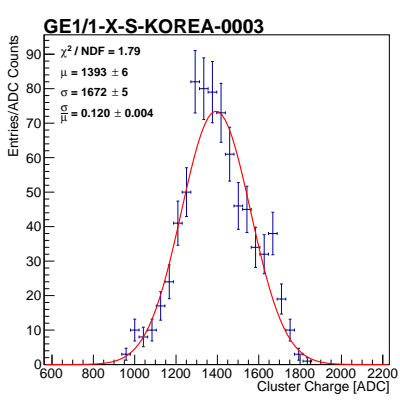

(d)

Figure 1: (a) The effective gas gain curves of the three GE1/1 detectors as a function of the voltage divider current. (b)-(d) The gain distributions of detector 1-3.

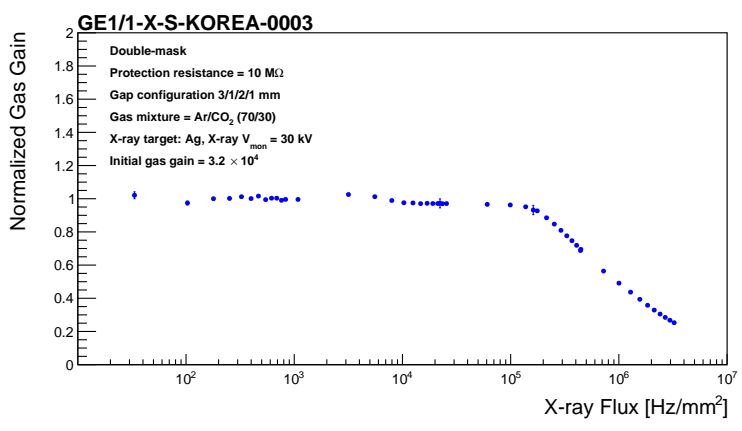

Figure 2: Normalized gas gain as a function of $\mathrm{x}$-ray flux.

gain uniformity of the detectors, the gains at 768 positions over the whole of the detectors were measured. Measured gains reached between $2 \times 10^{4}$ and $5 \times 10^{4}$ at $700 \mu \mathrm{m}$, the voltage divider current corresponding to the standard high voltage of the CMS GE1/1 detectors. Gain variances were measured from $10.2 \%$ to $16.2 \%$. The results are consistent with those of detectors using CERN produced foils.

The second measured property was the rate capability of the detector. The result is shown in Fig. 2. The gain remained stable for $\mathrm{x}$-ray fluxes up to $1 \times 10^{5} \mathrm{~Hz} \mathrm{~mm}^{-2}$. The measured capability is high enough for the CMS upgrade and other usual applications. The gain dropped for x-ray fluxes 


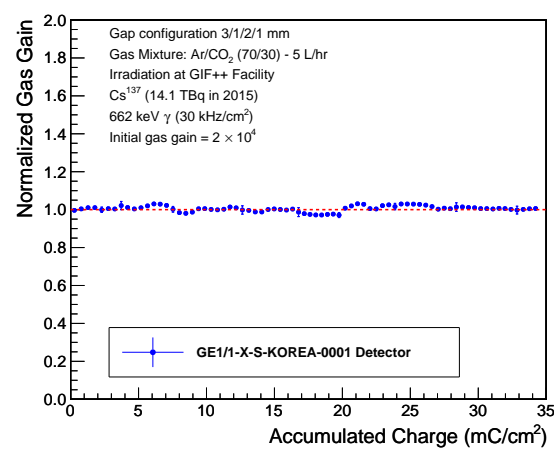

Figure 3: Normalized gas gain as a function of charge collected by readout.

over $1 \times 10^{5} \mathrm{~Hz} \mathrm{~mm}^{-2}$ because of the voltage drop at high protection resistance. When the detector was exposed to very high x-ray fluxes, the current flowing on GEM foils becomes significant, inducing a voltage drop at the resistance. Thus the voltage applied on GEM foils is reduced in this region.

The third measured property was the radiation hardness of the detector. One of the assembled detectors was installed in the new gamma irradiation facility (GIF++) [4] of CERN. The detector has been exposed to continuous x-ray irradiation from $14 \mathrm{TBq}$ of ${ }^{137} \mathrm{Cs}$ since March, 2018. As Fig. 3 shows, no effect of the classical aging was observed until collected readout charges up to $34 \mathrm{mCcm}^{-2}$. It corresponds to 113 (1.2) years of GE2/1 (ME0) detector use operating under HL-LHC conditions. Although much more charge should be collected for the Mecaro GEM foil validation for ME0 operation, current results are quite promising.

\section{Summary}

For the CMS phase-II upgrade, KCMS has made a consortium with Mecaro to become a second supplier of large size GEM foils. Mecaro produces large size GEM foils with the doublemask technique. The quality validation on the foils is ongoing by assembling CMS GE1/1 detectors with the Mecaro foils and measuring detector properties. The Mecaro foils are observed to work well in terms of the effective gas gain, the uniformity of the gain, and the rate capability and are found to be radiation hard.

\section{References}

[1] G. Apollinari et al., High-Luminosity Large Hadron Collider (HL-LHC): Technical Design Report V. 0.1, CERN, Geneva, 2017.

[2] CMS Collaboration, CMS Technical Design Report for the Muon Endcap GEM Upgrade, CERN-LHCC-2015-012. CMS-TDR-013.

[3] CMS Collaboration, The Phase-2 Upgrade of the CMS Muon Detectors, CERN-LHCC-2017-012. CMS-TDR-016.

[4] D. Pfeiffer et al., The radiation field in the Gamma Irradiation Facility GIF++ at CERN, Nucl. Instrum. Methods Phys. Res. A 866 (2017) 91 [physics.ins-det/161100299]. 